\title{
Informing Synthesis Routes with Correlated in Situ Electrothermal Studies in the TEM
}

Jenna Wardini ${ }^{1}$, William Bowman ${ }^{1}$, Jairo Gonzalez ${ }^{1}$ and George Harrington ${ }^{2}$

${ }^{1}$ University of California, Irvine, Irvine, California, United States, ${ }^{2}$ Kyushu University, Fukuoka, Kochi, Japan

The efficiency of solid oxide fuel cells (SOFCs) is limited by the performance of its primary components (i.e. the anode, cathode, and electrolyte). For instance, an effective SOFC cathode material must have high electronic and ionic conductivity, be porous to allow diffusion of oxygen gas into electrochemically active regions, and be chemically inert under operating conditions to prevent degraded performance over time [1].

Lanthanum strontium manganite (La1-xSrxMnO3, LSM) is a commonly used cathode material in SOFCs due to its high electronic conductivity and compatibility with the commonly used solid electrolyte yittriastabilized zirconia (YSZ). However, due to LSM's poor ionic conductivity at lower temperatures, its use is limited to high temperature $\left(800-1000{ }^{\circ} \mathrm{C}\right) \mathrm{SOFCs}$, where ions are more mobile. One proposed method of enhancing LSM's ion conductivity in the intermediate temperature range $\left(560-800{ }^{\circ} \mathrm{C}\right)$ is by reducing grain size to increase the relative volume of grain boundary with respect to grain interior. Grain boundaries in LSM have been shown to facilitate fast oxygen anion transport [2], enhancing ion conductivity. Unfortunately, grain boundaries also impede electron transport, so the optimal grain size is one that balances these outcomes. Optimizing materials for SOFC cathode performance continues to be an active area of research and one that can greatly benefit from correlated in situ electron microscopy studies.

Materials processing optimization problems like the one described above, are well-suited to an in situ approach that enables simultaneous observation of functional properties, morphology, crystal structure and chemistry. Using this information, processing routes can be modified to favor a structure and/or chemistry that results in the desired performance. Using this correlated approach, we explore the structural and chemical transformation of amorphous LSM films throughout sequential annealing steps while simultaneously monitoring film resistivity in air and vacuum. Rapid cooling of the LSM film freezes in the high-temperature state, enabling the development of a comprehensive characterization profile for each step of the annealing process through the use of analytical STEM techniques (EDS and direct-detection EELS) to probe chemical distribution, and (S)TEM imaging and electron diffraction to determine crystal structure. From this rich dataset, relationships between electrical behavior and microstructure can be established and used to tailor synthesis to favor a specific microstructural feature and resultant functional property.

In this presentation, we will present a range of correlated observations focused on linking LSM's electrical properties with its morphology and chemistry. For example, we observe that the perovskite LSM structure is unstable when annealed under low oxygen partial pressures, previously reported in bulk perovskite LSM systems [3], resulting in decomposition into multiple phases (Figure 1). Chemical mapping by EDS (Figure 2) shows the two most abundant phases, hexagonal La2O3 crystallites and a Mn-Sr-O amorphous film, quenched from its liquid state at $900{ }^{\circ} \mathrm{C}$ (confirmed by in situ heating video). 

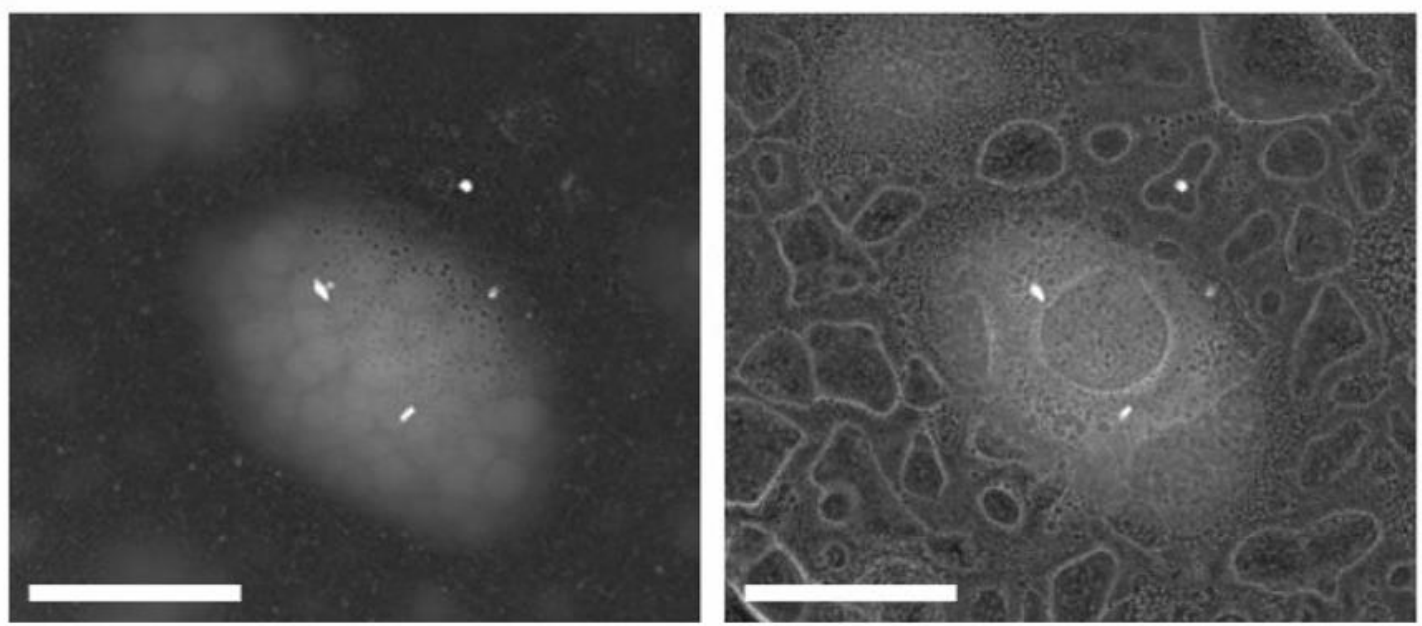

Figure 1. STEM-HAADF images of film before (left) and after (right) decomposition (scale bars are 0.2 $\mu \mathrm{m})$.

a)
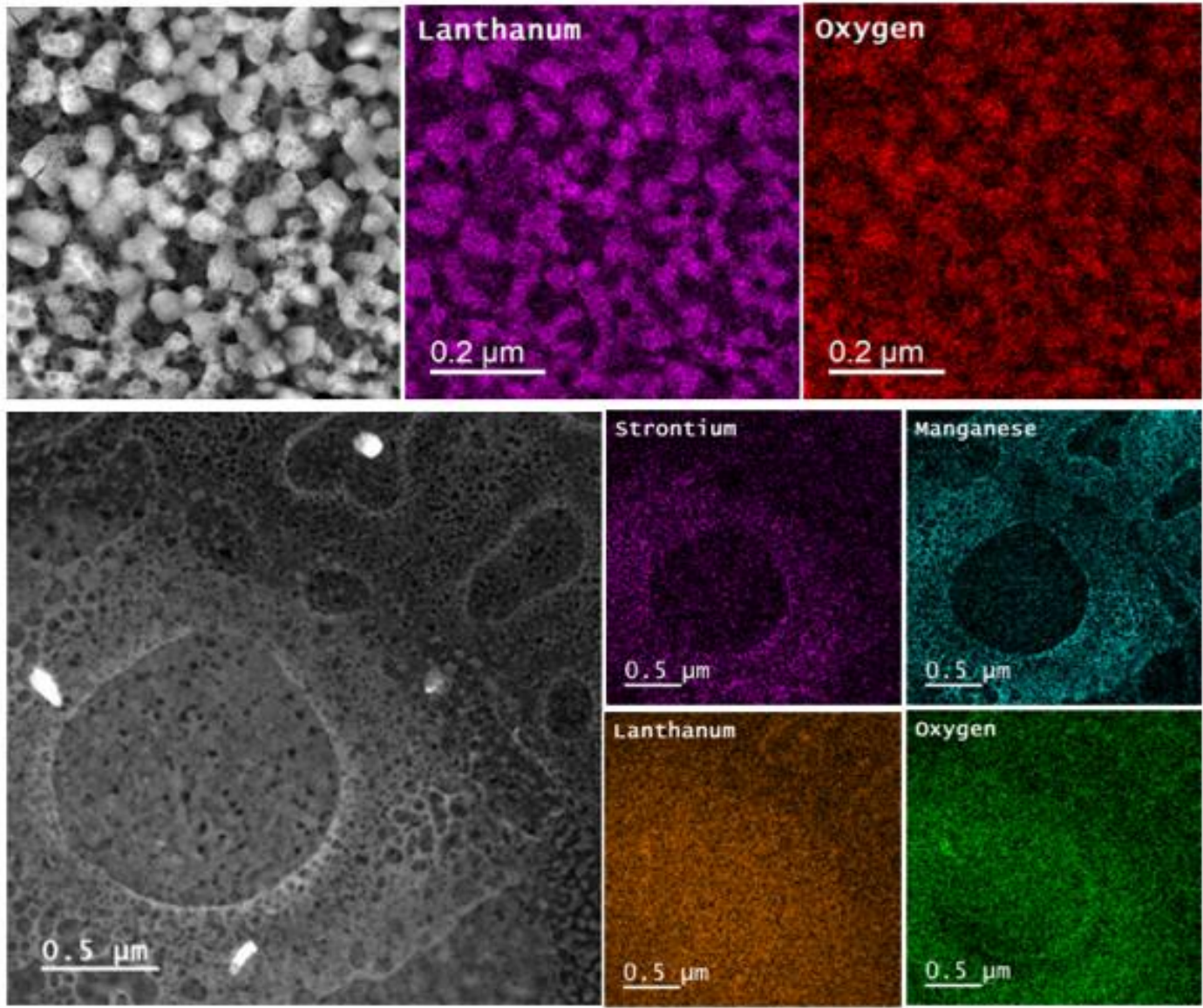

Figure 2. STEM-HAADF images and EDS maps demonstrating the formation of multiple phases including a) a hexagonal La2O3 and b) an amorphous $\mathrm{Mn}-\mathrm{Sr}-\mathrm{O}$, liquid at $>800{ }^{\circ} \mathrm{C}$ under vacuum annealing conditions. 


\section{References}

[1] Sun, Chunwen, Rob Hui, and Justin Roller. "Cathode materials for solid oxide fuel cells: a review." Journal of Solid State Electrochemistry 14.7 (2010): 1125-1144.

[2] Saranya, Aruppukottai M., et al. "Engineering mixed ionic electronic conduction in La0. 8Sr0. $2 \mathrm{MnO} 3+\delta$ nanostructures through fast grain boundary oxygen diffusivity." Advanced Energy Materials 5.11 (2015): 1500377.

[3] Cherepanov, V. A., L. Yu Barkhatova, and V. I. Voronin. "Phase equilibria in the La-Sr-Mn-O system." Journal of Solid State Chemistry 134.1 (1997): 38-44. 